\title{
O Que os Estudantes Consideram na Escolha do Curso de Graduação?
}

\author{
Maria Gabriela Cavalheiroㄹ; Caroline Antonelli Mendes²*; Ana Paula Carvalho Corrêa ${ }^{1}$; \\ Francielle Martins Ferreira1; Giédre Berretin-Felix²; Kelly Cristina Alves Silverio ${ }^{2}$ \\ ${ }^{1}$ Hospital de Reabilitação de Anomalias Craniofaciais da Universidade de São Paulo, Faculdade de Odontologia de \\ Bauru da Universidade de São Paulo \\ 2Programa de Pós-Graduação em Fonoaudiologia da Faculdade de Odontologia de Bauru da Universidade de São Paulo
}

*Autora para correspondência: caroline.mendes@usp.br

\section{RESUMO}

O objetivo do presente estudo é conhecer o perfil dos estudantes com interesse de ingressar em um curso de graduação, quanto à idade, nível socioeconômico e preferências de áreas de estudo. Participaram do estudo 101 estudantes do ensino médio e cursinho pré-vestibular com idades entre 14 e 44 anos (média de 18 anos) pertencentes a duas instituições de ensino públicas e três particulares do município de Bauru, Estado de São Paulo, a maioria estudantes do $3^{\circ}$ ano do ensino médio, com uma renda familiar entre dois e cinco salários mínimos e com preferência de estudo na área de Humanas. Os fatores que mais influenciam na escolha da profissão são: gostar da área de atuação, piso salarial e a afinidade com a disciplina no ensino médio. Na escolha do curso de graduação, os fatores que mais influenciam são: pertencer a uma instituição pública, se identificar com a área e o mercado de trabalho.

Palavras-Chave: Instituições Acadêmicas; Estudantes; Escolha da Profissão.

\begin{abstract}
The objective of the present study is to know the profile of students interested in joining one undergraduate course, regarding age, socioeconomic level and preferences of study areas. A total of 101 high school and pre-college students aged 14-44 years (mean age 18 years) belonging to two public institutions and three private institutions in the city of Bauru, State of São Paulo, participated in the study, most of them students of the 3rd year of high school, with a family income between two and five minimum wages and with a preference of study in the area of Humanities. The factors that most influence the choice of the profession are: to like the area of action, salary floor and the affinity with the discipline at high school. In choosing the undergraduate course, the factors that most influence are: belonging to a public institution, identifying with the area and the job market.
\end{abstract}

Keywords: Schools, Language and Hearing Sciences; Students; Career Choice.

\section{Introdução}

$\mathrm{Na}$ literatura, encontra-se como definição de escolha profissional a decisão do que fazer, de quem ser e a que lugar pertencer no mundo por meio do trabalho (BARDAGI et al., 2003). Essa escolha está envolvida com mudanças, perdas e medos de fracasso e desvalorização profissional (SOARES et al., 2007). Ela pode depender de vários fatores, como os políticos, econômicos, sociais, educacionais, familiares (ALMEIDA \& MELO-SILVA, 2011) e psicológicos. Os determinantes econômicos e sociais que influenciam as possibilidades de escolha profissional acabam por impossibilitar o indivíduo na sua busca do que seguir num curso universitário (VALORE \& CAVALLET, 2012; BASTOS, 2005).

Em relação aos fatores psicológicos, os interesses são as principais variáveis que podem influenciar essa escolha, seguidos pelas habilidades, os traços de personalidade, os valores, as expectativas com relação ao futuro e a maturidade para a escolha profissional (NEIVA et al., 2005). Estudos citam a influência da personalidade na escolha da profissão, apontando que estudantes tendem a escolher 
profissões que se adéquam mais ao seu tipo de personalidade; por exemplo, estudantes que têm uma personalidade mais curiosa e investigativa tendem a escolher áreas da ciência (MIHYEON, 2009; PORTER \& UMBACH, 2006). Muitos estudantes escolhem a profissão com base nas suas habilidades acadêmicas (BEGGS et al., 2008), outros a selecionam com base no retorno financeiro que a mesma proporciona, entretanto alguns estudantes ainda fazem a escolha profissional buscando realizar seus sonhos (MCGLYNN, 2007).

Conhecer e ter contato com profissionais da área de interesse podem também influenciar a escolha dos estudantes na escolha da profissão (ADAMSON, COVIC, KENCH \& LINCOLN, 2003; BRODSKY \& COOKE, 2000). O fato de a família possuir um negócio próprio também constitui um fator decisivo na escolha da profissão, pois gera nos estudantes o sentimento de obrigação de dar continuidade ao negócio da família, seguindo assim a área do negócio familiar (ZODY et al., 2006).

No Brasil, no que diz respeito à escolha profissional pelo aluno do ensino médio da rede pública de ensino, a necessidade de trabalhar, a falta de recursos para pagar um cursinho pré-vestibular ou uma faculdade e a impossibilidade de concorrer em igualdade com alunos oriundos de classes economicamente favorecidas podem influenciar de maneira decisiva as escolhas (BARTALOTTI \& MENEZES-FILHO, 2007; GUIGEN et al., 2014).

Até o ano de 2011, o Brasil possuía baixo índice de acesso à educação superior, quando comparado aos índices de outros países da América Latina (PAULA, 2011). Nesse contexto, foi aprovado o Plano Nacional de Educação (PNE), para vigorar entre 2011 e 2020, que prevê a ampliação do acesso a todos os níveis de educação, contando com o aumento de vagas e a democratização do acesso à educação em nível superior como algumas de suas metas (SCHNEIDER, 2012). Além da expansão das matrículas e da inclusão de jovens de baixa condição social, é imprescindível que lhes sejam assegurados os meios de permanência sustentável. Assim, acesso e permanência são aspectos essenciais do processo mais amplo de democratização da educação (DIAS SOBRINHO \& BRITO, 2008). Mesmo com políticas públicas a fim de facilitar o acesso e a permanência nos cursos de graduação, a taxa de evasão no ensino superior brasileiro chegou a 18,5\% no ano de 2007, sendo 12,4\% nas instituições públicas e $26 \%$ nas particulares (FRANCO, 2008).

Conhecer as motivações e o perfil dos alunos que irão ingressar no ensino superior se faz importante para favorecer a relação estabelecida entre $o$ estudante e a instituição de ensino, garantir a autonomia do aluno no envolvimento com atividades curriculares e extracurriculares da graduação e alcançar o sucesso no processo de ensino-aprendizagem na graduação.

Dessa forma, o objetivo do presente estudo é conhecer o perfil dos estudantes com interesse de ingressar em um curso de graduação, quanto à idade, ao nível socioeconômico e às preferências de áreas de estudo.

\section{Métodos}

O estudo transversal e observacional foi conduzido de acordo com a Resolução 196/96 do Conep e aprovado pelo Comitê de Ética em Pesquisas com seres humanos da instituição (FOB/USP 004/2012) de origem. A concordância para a participação nesta pesquisa se deu por meio da assinatura do Termo de Consentimento Livre e Esclarecido (TCLE), realizada pelos pais dos alunos que eram menores de idade ou pelo próprio participante, caso fosse maior de dezoito anos.

Participaram do estudo 101 estudantes do ensino médio e cursinho pré-vestibular com idades entre catorze e 44 anos (média de dezoito anos), pertencentes a duas instituições públicas e três particulares do município de Bauru, estado de São Paulo.

A coleta de dados se deu por meio da aplicação de um questionário elaborado pelos pesquisadores e composto por dezesseis questões, sendo quatro abertas e doze de múltipla escolha, quanto aos dados de identificação, perfil socioeconômico, preferências de áreas de estudo e fatores que influenciam a escolha da profissão e do curso de graduação. Para a apresentação dos resultados da primeira e 
segunda etapa, recorreu-se à análise estatística descritiva por meio de porcentagem quanto à idade, escolaridade, nível socioeconômico, preferências de áreas de estudo, fatores que influenciam na escolha da profissão e curso de graduação dos estudantes.

\section{Resultados}

A maior parte da população estudada foi de estudantes que cursavam o terceiro ano do ensino médio, o que pode ser visualizado na Figura 1.

Com relação à análise do nível socioeconômico, a maioria dos estudantes entrevistados declarou possuir renda familiar de dois a cinco saláriosmínimos. Cabe ressaltar que alguns participantes da pesquisa deixaram essa questão sem resposta (Figura 2).

Quanto às preferências de área de estudos, foi possível observar que 48,7\% dos entrevistados preferem a área de Humanas, já 29,2\% optaram por Exatas e 22,1\% preferem a área de Biológicas. Nessa questão alguns participantes assinalaram mais de uma alternativa.

Em relação aos fatores que interferem na escolha profissional, os estudantes consideram: "gostar da área de atuação", o salário e a afinidade com a área no ensino médio. Outros fatores podem ser observados na Figura 3.

Quanto aos fatores que interferem na escolha do curso, os mais citados pelos estudantes foram: a faculdade pertencer a uma instituição pública, gostar da área de atuação e apresentar bom mercado de trabalho. Outros fatores podem ser observados na Figura 4.

\section{Discussão}

O estudo demonstrou que a maior parte dos participantes frequentava o terceiro ano do ensino médio, depois vinham os alunos do curso pré-vestibular (Figura 1). Esses estudantes representam a população que participa dos processos seletivos para ingressar no curso de graduação das grandes universidades do país. Assim, a escolha profissional é uma questão delicada quando o estudante não dispõe de informações suficientes sobre a futura profissão. Outro aspecto diretamente ligado a essa decisão é a idade dos estudantes, que nesse estudo apresenta uma média de dezoito anos. Nessa faixa etária, há grande dúvida sobre a escolha profissional, o que torna a decisão mais

\section{Perfil de Escolaridade dos Estudantes do Ensino Médio}

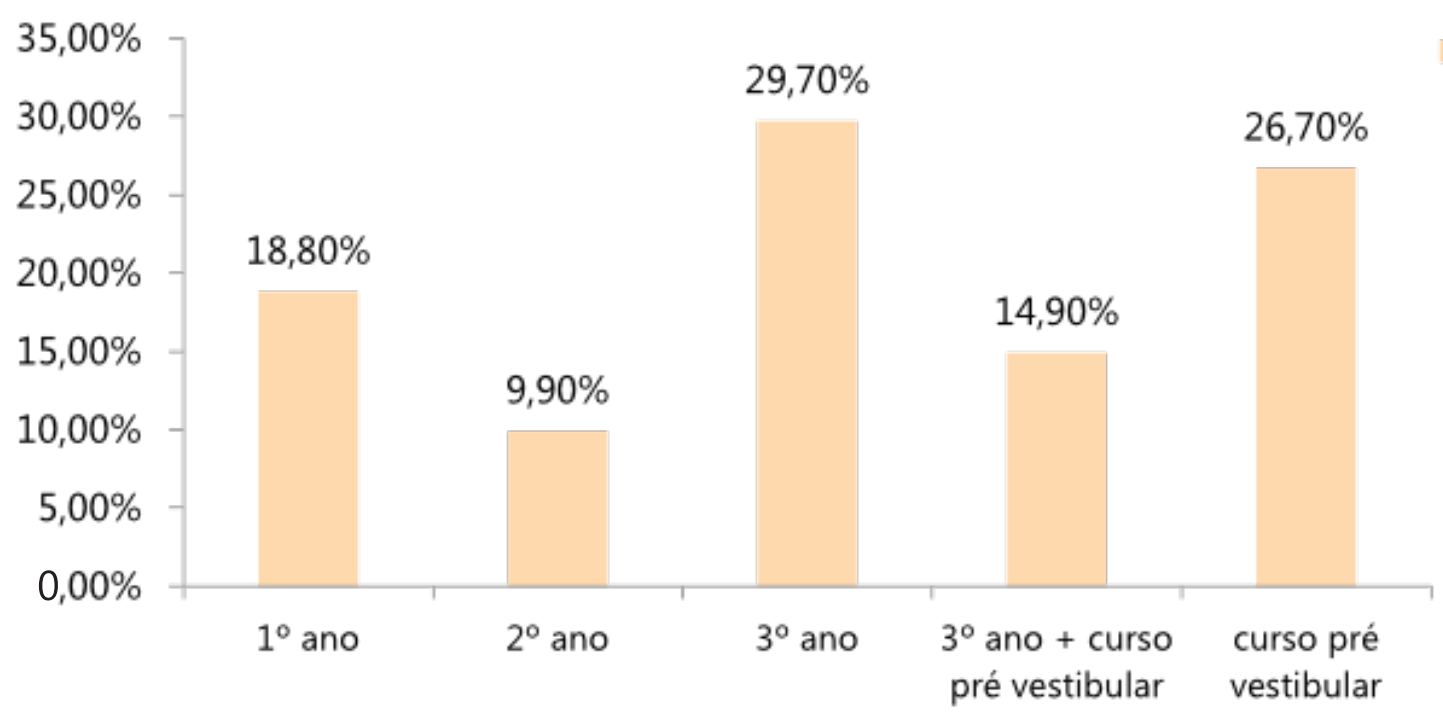

Figura 1 - Perfil dos estudantes da amostra em relação à escolaridade. 


\section{Nível socioeconômico: Renda dos estudantes}

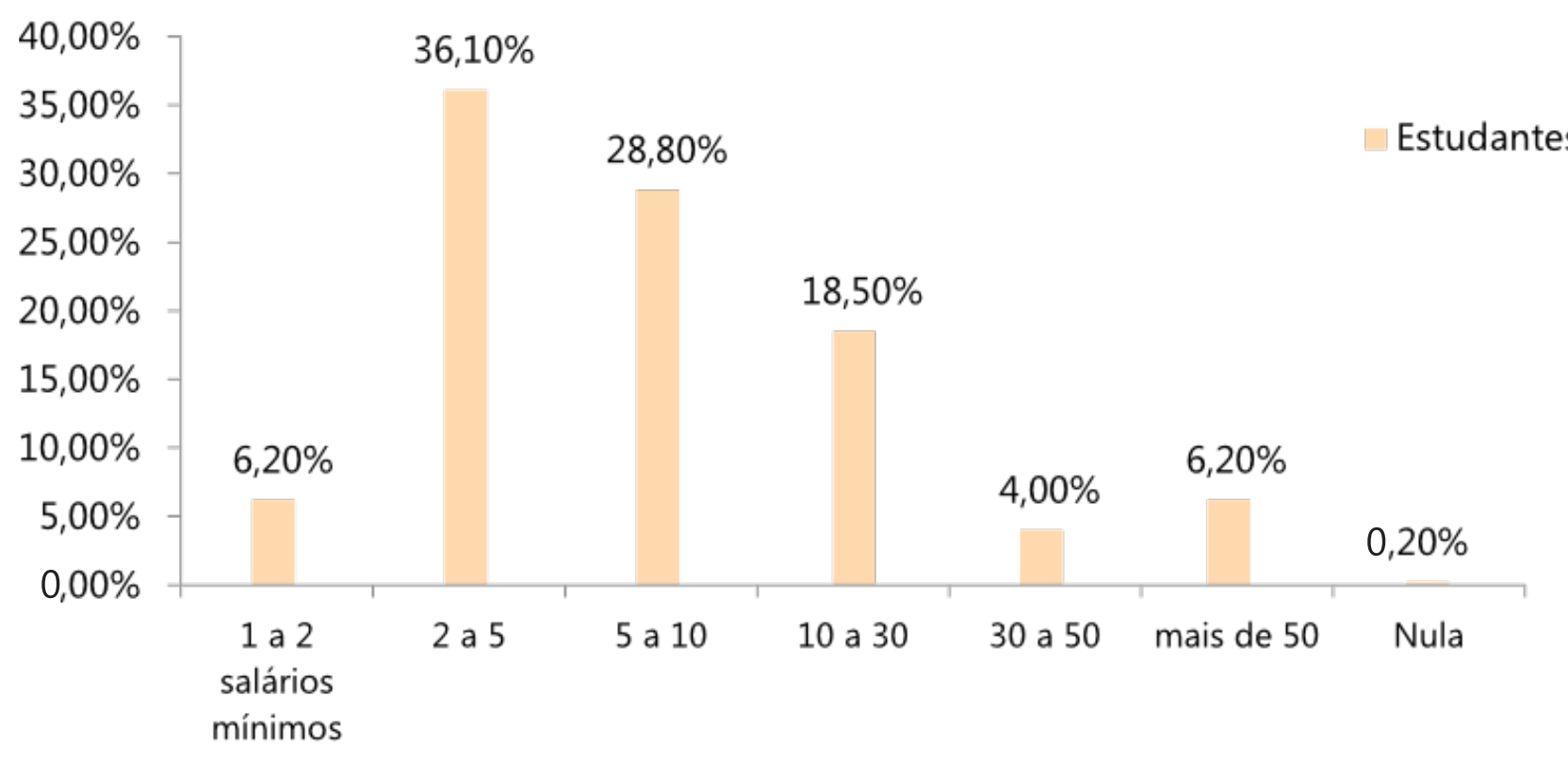

Figura 2 - Distribuição de estudantes de acordo com o nível socioeconômico declarado.

\section{Fatores que influenciam a escolha profissional}

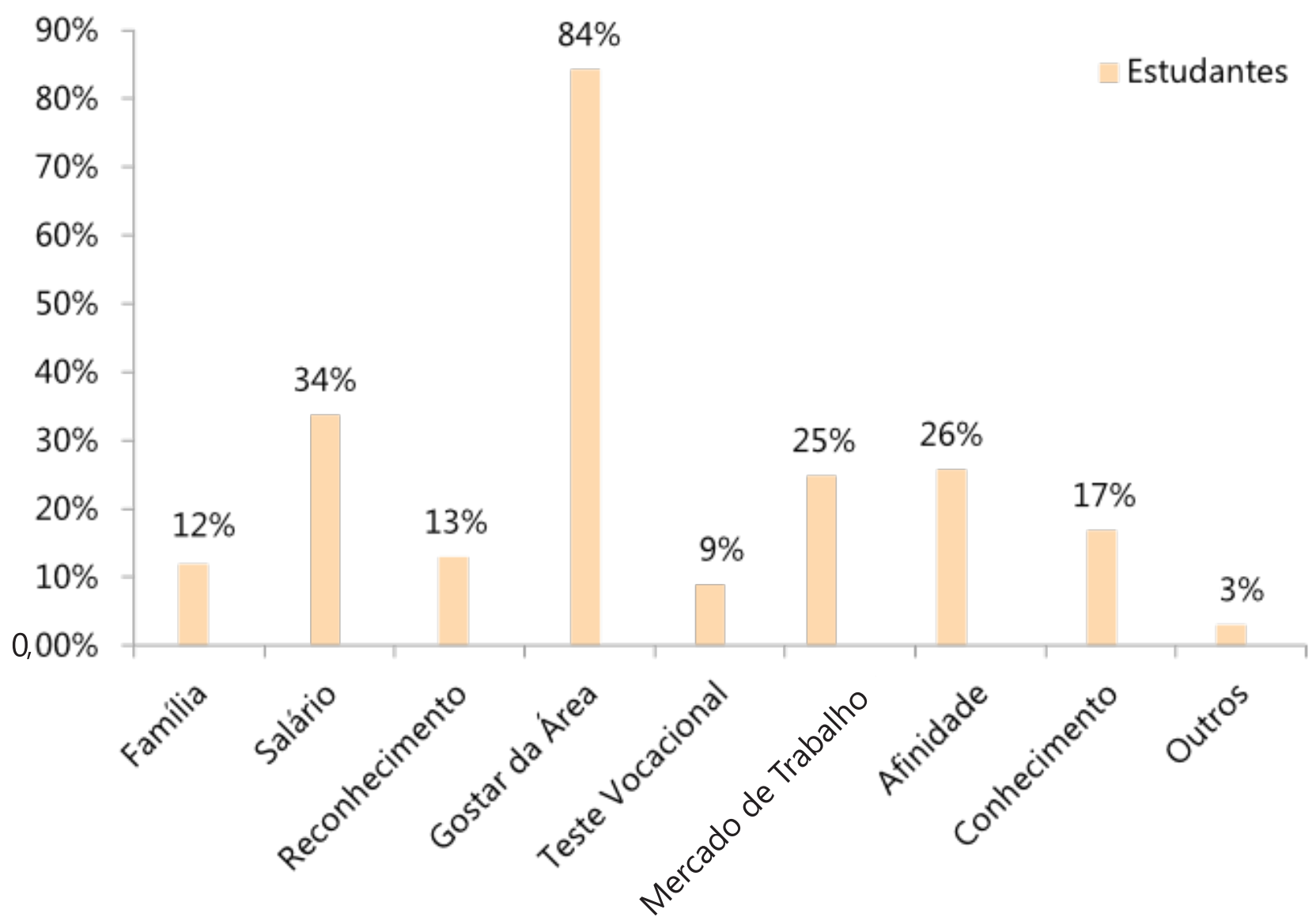

Figura 3 - Distribuição de estudantes de acordo com fatores que influenciam a escolha profissional. 


\section{Fatores que interferem na escolha do curso}

Estudantes

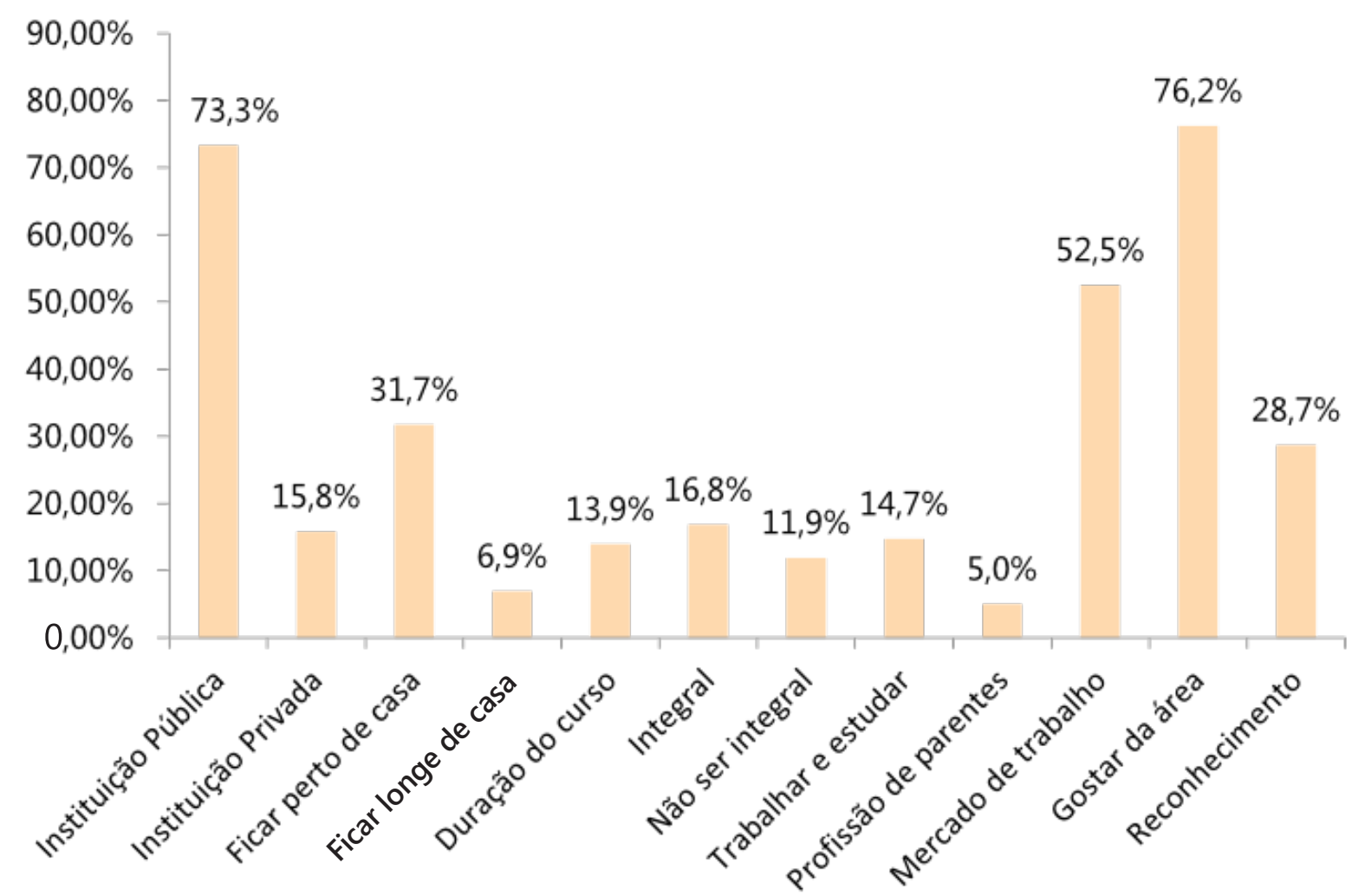

Figura 4 - Distribuição de estudantes de acordo com fatores que influenciam a escolha do curso.

difícil, visto que os estudantes estão passando por uma fase transitória entre a adolescência e a vida adulta e podem sentir insegurança emocional, assim como falta de experiência para definir a profissão (GOMES \& MALACARNE, 2009).

Segundo um estudo demográfico realizado pelo Instituto Brasileiro de Geografia e Estatística, em 2012, 15,3\% das mulheres entre 25 e 29 anos possuíam curso superior, enquanto os homens, nesse mesmo enquadramento, representavam 10,6\%. $\mathrm{O}$ questionário utilizado não contemplava o item gênero, portanto não foi possível analisar essa variável. No entanto, podem aparecer diferenças quanto à motivação, às expectativas e aos níveis de autonomia na escolha do curso em grupos de alunos conforme gêneros, cursos e semestres (ALMEIDA \& SOARES, 2003).

A renda socioeconômica foi analisada no presente estudo, sendo que a maioria dos estudantes respondeu apresentar uma renda entre dois e cinco salários-mínimos (Figura 2). De acordo com pesquisadores, embora a sociedade ofereça oportunidades educacionais e profissionais para todos os jovens, os de baixa renda se encontram em desvantagem quanto às informações e à metodologia de escolha da profissão (BACHA \& OSÓRIO, 2004). Os estudantes que dispõem de maior renda familiar consequentemente têm maiores e melhores condições de acesso a informações sobre as diferentes profissões da atualidade, encontrando maior facilidade no momento da escolha da profissão.

O questionário utilizado na metodologia buscou traçar também os fatores que interferem na escolha profissional. A maioria da população citou "gostar da área de atuação" como o fator que mais influencia no momento de optar por qual profissão seguir. Outros fatores foram mencionados, como o nível salarial, a afinidade com disciplinas do ensino médio, as condições do mercado de 
trabalho para a profissão e a atuação profissional (Figura 3). Ao escolher a área de preferência para seguir a profissão, entre as opções listadas estavam as áreas de Humanas, Biológicas e Exatas. Nesse estudo a maioria dos estudantes optou pela área de Humanas. O estudante busca associar as disciplinas de sua preferência que fazem parte da grade curricular no ensino médio com a futura profissão.

Cabe ressaltar outros aspectos que se relacionam com essa escolha, como a habilidade de reconhecer as próprias preferências e os aspectos emocionais, uma vez que os estudantes enfrentam sentimentos de insegurança, medo e expectativas quanto ao futuro, podendo estes ser intensificados pelos sentimentos dos familiares, o que torna, de certa forma, a situação ainda mais complexa (ELY et al., 2010).

Além de escolher a profissão, o estudante deve optar pelo curso, ou seja, a ferramenta por meio da qual os conhecimentos necessários para exercer uma profissão são obtidos. Quanto à escolha do curso, os fatores mais relatados como influentes na decisão foram: pertencer a uma instituição pública, identificar-se com a área e o mercado de trabalho, o que coincide com estudos anteriores que apontam a preferência por uma profissão na área que mais se adequa à personalidade (MIHYEON, 2009; PORTER \& UMBACH, 2006). Outros fatores citados, mas com menor frequência, foram: estudar perto de casa, o curso apresentar reconhecimento profissional, poder conciliar trabalho e estudo, entre outros (Figura 4).

Não foram encontrados estudos que indiquem influência, na escolha da profissão, do fato de a instituição de ensino ser pública ou privada, como apreendido nesta pesquisa; sabe-se, entretanto, que o interesse pelas instituições públicas se deve ao fato de as universidades públicas serem reconhecidas pela referência de ensino de qualidade, algumas com destaque internacional, e pela quantidade de estudantes que elas atraem para as cidades em que se localizam, sendo que muitos cursos apresentam elevada concorrência (RIGHI \& RUPPENTHAL, 2013). Em estudo com alunos de baixa renda aponta-se que o trabalho e, consequentemente, a escolha da profissão são fatores que estão relacionados, uma vez que há a possibilidade de compensação financeira (BARTALOTTI \& MENEZES-FILHO, 2007).

Dessa forma, o perfil dos alunos com interesse de ingressar em um curso de graduação se faz de jovens com preocupações a respeito do salário, do tipo de instituição de ensino e de como satisfazer o mercado de trabalho, mas que se sentem motivados a atuar em suas áreas de afinidade. A motivação é um dos fatores mais importantes para alcançar o aprendizado (BZUNECK, 2004); no entanto, existem obstáculos durante o contexto acadêmico que podem prejudicá-la e afetar o desempenho acadêmico e aspectos psicossociais do aluno, como questões pessoais, exigências acadêmicas que devem ser desempenhadas com eficiência e a adaptação a um novo ambiente (ALMEIDA \& SOARES, 2003). O processo de adaptação ao ensino superior e o rendimento acadêmico (BZUNECK, 2004; CUNHA \& CARRILHO, 2005), bem como, nesse momento, as políticas de permanência e o preparo dos docentes e da equipe da graduação se fazem necessários para auxiliar no processo de adaptação do aluno.

\section{Conclusão}

Foi possível constatar que a população da pesquisa apresentou média de dezoito anos de idade, a maioria estudantes do terceiro ano do ensino médio, com uma renda familiar entre dois e cinco salários-mínimos e com preferência de estudo na área de Humanas. Os fatores que mais influenciam na escolha da profissão são: gostar da área de atuação, piso salarial e a afinidade com a disciplina do ensino médio. $\mathrm{Na}$ escolha do curso de graduação, os fatores que mais influenciam são: pertencer a uma instituição pública e identificar-se com a área e o mercado de trabalho.

\section{Referências Bibliográficas}

ADAMSON, B.; COVIC, T.; KENCH, P. \& LINCOLN, M. "Determinants of Undergraduate Program Choice in Two Health Science Fields: Does Personality Influence Career Choice?". Focus on Health Professional Education, vol. 5, n. 2, 2003, pp. 34-47. 
ALMEIDA, F. H. \& MELO-SILVA, L. L. "Influência dos Pais no Processo de Escolha Profissional dos Filhos: uma Revisão da Literatura". Psico USF, vol. 16, n. 1, 2011, pp. 75-85.

ALMEIDA, L. S. \& SOARES, A. P. "Os Estudantes Universitários: Sucesso Escolar e Desenvolvimento Psicossocial". In: MERCURI, E. \& POLYDORO, S. A. J. (orgs.). Estudante Universitário: Características e Experiências de Formação. Taubaté, SP: Cabral, 2003, pp. 15-40.

BACHA, S. M. C. \& OSÓRIO, A. M. N. "Fonoaudiologia \& educação: uma revisão da prática histórica". Rev Cefac., vol. 6, n. 2, 2004, pp. 215-21.

BARDAGI, M. P.; LASSANCE, M. G. P. \& PARADISO, A. C. "Trajetória Acadêmica e Satisfação com a Escolha Profissional de Universitários em Meio de Curso". Rev Bras Orientac Prof., vols. 4, n. 1-2, 2003, pp. 153-66.

BARTALOTTI, O. \& MENEZES-FILHO, N. "A Relação entre o Desempenho da Carreira no Mercado de Trabalho e a Escolha Profissional dos Jovens". Econ Aplic., vol. 11, n. 4, 2007, pp. 487-505.

BASTOS, J. C. "Efetivação de Escolhas Profissionais de Jovens Oriundos do Ensino Público: um Olhar sobre Suas Trajetórias". Rev. Bras. Orientac. Prof., vol. 6, n. 2, 2005, pp. 31-43.

BEGGS, J. M.; BANTHAM, J. H. \& TAYLOR, S. "Distinguishing the Factors Influencing College Students' Choice of Major". College Student Journal, vol. 42, n. 2, 2008, p. 381.

BRODSKY, M. \& COOKE, P. "Influences in the Decision Making Process for Careers as a SpeechLanguage Pathologist or an Audiologist". Journal of Employment Counseling, vol. 37, n. 3, 2000, pp. 178-89.

BZUNECK,J. A. "A Motivação do Aluno Orientado a Metas de Realização". In: BORUCHOVITCH, E. \& BZUNECK, J. A. (orgs.). A Motivação do Aluno: Contribuições da Psicologia Contemporânea. Petrópolis, RJ: Vozes, 2004, pp. 58-77.

CUNHA, S. M. \& CARRILHO, D. M. "O Processo de Adaptação ao Ensino Superior e o Rendimento Acadêmico". Psicologia Escolar e Educacional, vol. 9, n. 2, 2005, pp. 215-224.

DIAS SOBRINHO, J. \& BRITO, M. R. F. "La Educación Superior en Brasil: Principales Tendencias y Desafíos". Avaliação, Rev. da Av. da Educ. Sup., vol. 13, n. 2, 2008, pp. 487-507.

ELY, A.; TEIXEIRA, R. F. \& MARQUARDT, S. E. L. "Determinantes da Escolha Profissional em Estudantes da Periferia Urbana: um Estudo de Caso na Cidade de Criciúma-SC". Anais do II Seminário de Ciências Sociais Aplicadas, Criciúma, 2010.

FRANCO, A. P. "Ensino Superior no Brasil: Cenário, Avanços e Contradições". Jornal de Politicas Educacionais, n. 4, 2008, pp. 53-63.
GOMES, A. R. C. G. \& MALACARNE, V. "Os Alunos do Ensino Médio e os Desafios das Escolhas para a Formação Profissional", 2009. Disponível em <http://www.diaadiaeducacao.pr.gov.br/portals/pde/ arquivos/2419-8.pd† $>$. Acessado em: 15 dez. 2013.

GUIGEN, A. P.; ZABEU, J. S.; FREIRE, T.; CAMPOS, P. D.; FELIX, G. B. \& FERRARI, D. V. "Fonoaudiologia como Opção de Carreira Universitária: Estudo Exploratório". Rev Cefac., vol. 16, n. 3, 2014, pp. 974-84.

MCGLYNN, A. P. "Achieving the Dream: What is It, and What's New?". The Hispanic Outlook in Higher Education, vol. 18, n. 4, 2007, pp. 44-5.

MIHYEON, K. 2009. The Relationship between Thinking Style Differences and Career Choice for High-Achieving High School Students. PhD Diss., Dept of Education, The College of William and Mary, United States, Virginia.

NEIVA, K. M. C.; SILVA, M. B.; MIRANDA, V. R. \& ESTEVES, C. "Um Estudo sobre a Maturidade para a Escolha Profissional de Alunos do Ensino Médio". Rev. Bras. Orientac. Prof., vol. 6, n. 1, 2005, pp. 1-14.

PAULA, M. F. C. "Educação Superior e Inclusão Social na América Latina: um Estudo Comparado entre Brasil e Argentina". In: PAULA, M. F. C. \& FERNÁNDEZ-LAMARRA, N. Reformas e Democratização da Educaşão Superior no Brasile na América Latina. Aparecida, SP: Ideias \& Letras, 2011, pp. 53-96.

PORTER, S. R. \& UMBACH, P. D. "College Major Choice: An Analysis of Person-Environment". Research in Higher Education, vol. 47, n. 4, 2006, pp. 429-49.

RIGHI, M. L. \& RUPPENTHAL, J. E. "A Influência de uma Universidade na Geração de um Polo Regional de Ensino Superior". ABCustos, vol. 8, n. 1, 2013, pp. 68-83.

SCHNEIDER, G. S. G. Políticas Públicas de Educaşão: uma Reflexão a Partir do novo Plano Nacional de Educasão. Dissertação (Mestrado em Pedagogia), Brasília (DF), Faculdade de Educação, Universidade de Brasília, 2012.

SOARES, D. H. P.; KRAWULSKI, E.; DIAS, M. S. L. \& D'AVILA, T. G. "Orientação Profissional em Contexto Coletivo: uma Experiência em Pré-Vestibular Popular". Psicol Cienc Prof., vol. 27, n. 4, 2007, pp. 746-59.

VALORE, L. A. \& CAVALLET, L. H. R. "Escolha e Orientação Profissional de Estudantes de Curso Pré-Vestibular Popular". Psicol Soc., vol. 24, n. 2, 2012, pp. 354-63.

ZODY, Z. S.; MacDERMID, S.; SCHRANK, H. \& SPRENKLE, D. "Boundaries and the Functioning of Family and Business Systems". Journal of Family and Economic Issues, vol. 27, n. 2, 2006, pp. 185-206.

Publicado em 04/07/2018. 